\title{
Effect of Enzymic Hydrolysis of the Scissile Bond on the Secondary Structure of a Dimeric Globular Protein
}

\author{
Tomoko KomiYama, Takayasu MoRI, Hajime OMATA \\ and Makoto MIWA \\ Department of Industrial Chemistry, Seikei University, \\ Musashino, Tokyo 180, Japan
}

(Received April 25, 1985)

\begin{abstract}
In order to examine the effect of enzymic cleavage of the reactive site on the secondary structure of the globular protein, Streptomyces subtilisin inhibitor, we studied the thermal denaturation of the moderated inhibitor (the scissile bond cleaved) by means of $\mathrm{CD}$ spectroscopy and compared the thermal stability with that of the intact protein. The cleavage gave a significant effect on the thermal stability and lowered the denaturation temperature to $62^{\circ} \mathrm{C}, 20^{\circ} \mathrm{C}$ below that observed for the intact inhibitor. However, the mechanism of thermal denaturation of the moderated inhibitor was similar to that of the intact one. Based on the hydrophobic and hydrophilic tendency of side chains of amino acid residues, we discuss the effect of hydrolysis at the scissile bond of the protein in the outer flexible region and the $\alpha$-helix and its neighborhood.

KEY WORDS Nicked Inhibitor / Thermal Denaturation / Circular Dichroism / Conformation / Hydropathy /
\end{abstract}

Streptomyces subtilisin inhibitor (SSI) is a dimeric globular protein, whose subunit has a single polypeptide chain of 113 residues and two intrachain disulfide bonds. ${ }^{1-3}$ Several domestic research groups have cooperatively studied this inhibitor from various standpoints ${ }^{4}$ since its isolation from Streptomyces albogriseolus by Murao and Sato. ${ }^{5}$ Two subunits are in contact with each other to form the hydrophobic core of the dimeric protein with extensive $\beta$-sheet strands. ${ }^{3,6}$ SSI has an unusual stability against thermal ${ }^{7,8}$ and acid $^{9,10}$ denaturation. Microbial alkaline proteases such as subtilisin $\mathrm{BPN}^{\prime}$ hydrolyze the reactive site (Met 73-Val 74) under a particular condition and give the nicked SSI which is abbreviated as $\mathrm{SSI}^{*}{ }^{11,12} \mathrm{SSI}^{*}$ is a reaction intermediate formed in the protein-protein interaction. Since the reactive site is located in a cyclic peptide bonds linked by a disulfide bond $(\text { Cys } 71-\text { Cys } 101)^{13}$, the modification of SSI leaves the protein molecule in one unit as schematically shown in Figure 1 drawn based on the X-ray study. ${ }^{3}$ Denaturation of SSI* has

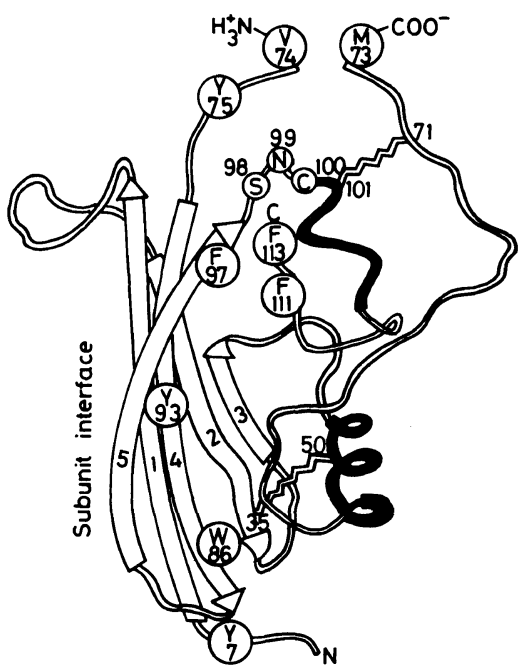

Figure 1. Schematic drawing of the structure of SSI*. Winding dark lines and arrows indicate $\alpha$-helices and $\beta$ strands, respectively. Zigzag lines represent two disulfide bonds (Cys 35-Cys 50 and Cys 71-Cys 101). 
not been studied except for a preliminary fluorometric study on the environment of a Trp residue in the subunit. ${ }^{14}$ To examine the effect of the cleavage on the secondary structure, we studied the thermal denaturation of SSI* with CD spectroscopy in detail and compared the denaturation temperature, $\Delta H$ and unfolding process with those of the intact SSI. Difference CD of SSI* relative to that of SSI indicated the $\alpha_{2}$-helix region and its neighborhood to be loosen by hydrolysis of the reactive site. Based on the relative hydropathy scale of amino acid side chains proposed by Kyte and Doolittle, ${ }^{15}$ we discuss the experimental findings. We found SSI* to be much less stable than SSI.

\section{EXPERIMENTAL}

\section{Materials}

Crude SSI kindly provided by Prof. S. Murao and his coworkers of University of Osaka Prefecture, was purified by an ordinary method. ${ }^{16}$ The purified enzyme (subtilisin $\mathrm{BPN}^{\prime}$ ) and a small quantity of authentic sample was a gift from Prof. B. Tonomura of Kyoto University. All other reagents were the best grade available.

Preparation of SSI*. SSI* was prepared by destroying the outer enzyme portion of the complex formed between SSI and the enzyme at the solution $\mathrm{pH} 2.5$ and $0^{\circ} \mathrm{C}$. This procedure was developed by. Matsumori et al. ${ }^{11}$ and Iwanari. $^{12}$ To isolate and purify the SSI* fraction, the solution was applied to ion exchange chromatography on QAE-Sephadex. To identify the nicked bond, we reduced the SSI* fraction with excess dithioerythritol (DTE) and blocked the produced thiol groups by $N$-(1-anilinonaphthyl-4)-maleimide (ANM) in vacuo following the previously described method. ${ }^{6}$ ANM reacts selectively with the thiol groups and turns fluorescent. ${ }^{17}$ The reaction mixture was subjected to gel-filtration on a Sephadex G-100 column. Denaturation of an authentic sample of SSI* presented from Prof.
B. Tonomura was also examined to identify our SSI* sample.

\section{Measurements}

CD spectra were taken with a JASCO J-40 circular dichroism spectrophotometer. The optical length of the water-jacketed cell and protein concentration were $1 \mathrm{~mm}$ and 0.125 $\mathrm{mg} \mathrm{ml}^{-1}\left(5.4 \times 10^{-6}\right.$ in $0.05 \mathrm{M}$ phosphate buffer ( $\mathrm{pH} 7.0, \mu=0.1 \mathrm{NaCl}$ ), respectively. The temperature was controlled between $20^{\circ} \mathrm{C}$ and $70^{\circ} \mathrm{C}$ by circulating thermostatted water into the cell jacket, and monitored by thermocouples fixed at the cell surface. We adjusted the heating rate to about $0.2^{\circ} \mathrm{C} \mathrm{min}^{-1}$ and used $30 \mathrm{~min}$ for one scan of a CD measurement at a certain temperature, although the temperature profile of SSI* CD showed scarecely any temperature dependence.

Analysis of CD Spectra. CONTIN program provided by Provencher ${ }^{18}$ was applied to analyze the CD spectrum of SSI*. This CD analysis is based on a flexible least square method and classifies the secondary structure as $\alpha$ helix, $\beta$-sheet, and the remainder. The class of the remainder includes $\beta$-turn, random coils, and nonpeptidic chromophores.

\section{RESULTS AND DISCUSSION}

\section{Identification of SSI*}

SSI* was fully reduced with ten times excess (on SSI dimer basis) of DTE in vacuo. The produced thiol groups were labeled with ANM and were quantitatively analyzed from fluorescence intensity of ANM complexed with the thiol groups. Figure 2 shows the elution pattern on a Sephadex G-100 column of the reaction mixture. Peaks $L$ and $S$ show intense fluorescence at $460 \mathrm{~nm}$ and a little CD in the far-UV region. These peaks suggest two peptide chains with different molecular weights resulting from complete reduction of SSI*. The peak $\mathrm{N}$ shows no $\mathrm{CD}$ in the far-UV region, indicating that it is nonpeptidic, although it shows similar fluorescence at $460 \mathrm{~nm}$. 


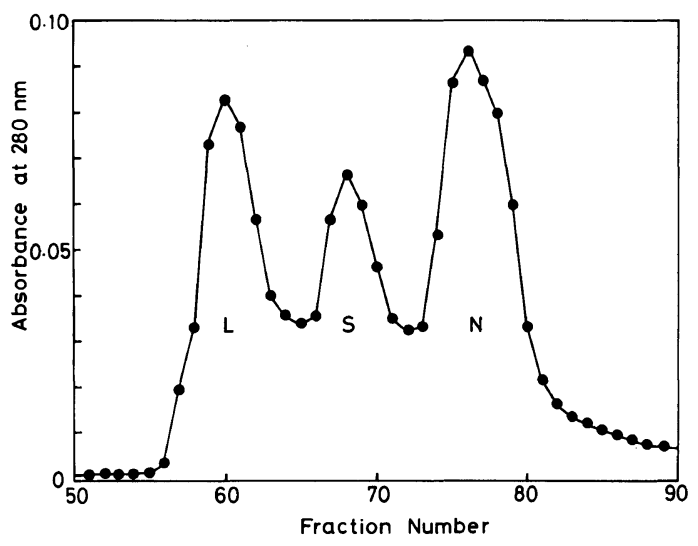

Figure 2. Elution pattern of the completely reduced SSI* solution through Sephadex G-100. The column $(1.6 \times 70 \mathrm{~cm})$ was eluted with a mixture of acetone, ethanol and $0.05 \mathrm{M}$ phosphate buffer of $\mathrm{pH} 7$ (4.5:4.5: $1.0, \mathrm{v} / \mathrm{v})$, and $2 \mathrm{ml}$ fractions were collected.

We estimated the ratio of the ANM group number in a peptide chain $L$ to that of $S$ to be three on the basis of the molar extinction coefficient and fluorescence intensity of the ANM complexed with thiol groups. These results show that complete reduction of the prepared SSI* produces two peptide chains, the longer one with three thiol groups and the shorter one with one thiol group. These results are consistent with the fact that the nicked bond is in the loop of the second disulfide bond as shown in Figure $1 .^{13}$ An elution profile of the native SSI* on a Sephadex G-100 column corresponded to that of the SSI on the same column. Furthermore, the thermal denaturation of SSI* authentic sample was examined and the same temperature profile of CD spectra was obtained. These facts show the prepared SSI* sample to be genuine.

\section{Difference CD between SSI* and SSI}

Figure 3 shows CD spectra of SSI* and SSI. The mean residue molar ellipticities at $220 \mathrm{~nm}$ are -7370 and -9170 for SSI* and SSI, respectively. Difference $\mathrm{CD}$ of $\mathrm{SSI}^{*}$ relative to that of SSI (dotted line in Figure 3) shows two

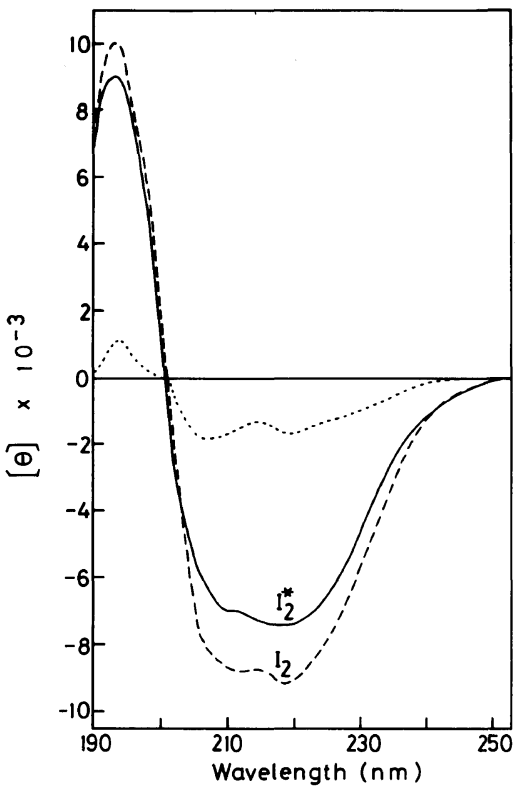

Figure 3. CD spectra of SSI* (solid line) and SSI (dashed line) and the difference CD (dotted line) between them at $20^{\circ} \mathrm{C}$. Protein concentration was $1 \mathrm{mg} \mathrm{ml}^{-1}$ $\left(4.4 \times 10^{-5} \mathrm{M}\right)$ in $0.05 \mathrm{M}$ phosphate buffer $(\mathrm{pH} 7, \mu=0.1$ $\mathrm{NaCl})$. Optical length of the cell was $0.1 \mathrm{~mm}$.

negative peaks at $207 \mathrm{~nm}$ and $220 \mathrm{~nm}$ characteristic of $\alpha$-helix, indicating the unfolding of the $\alpha$-helix moiety resulted from hydrolysis of the scissile bond. The fractional content of $\alpha$-helix and $\beta$-sheet of SSI* was estimated to be $23 \%$ and $43 \%$, respectively. SSI have been estimated to contain $24 \% \alpha$-helix and $36 \% \beta$ sheet $^{6,8}$ by this method. Any other methods gave inconsistent plots with the measured CD spectrum of SSI. However, this CD analysis contradicts the above difference CD. A characteristic of this method is that the molar ellipticities of $\alpha$-helix, $\beta$-sheet and the remainder vary according to the protein. We notice that significant quantities obtained from this method are the values of relative ellipticities of the $\alpha$-helix and $\beta$-sheet rather than fractional content. The mean residue ellipticities [ $\theta$ ] of the $\alpha$-helix of SSI* reduces to about $79 \%$ that of SSI. The analysis function of $\beta$ - 
sheet was almost identical for both proteins. This means that the hydrolysis cleavage at the scissile bond profoundly affects the $\alpha$-helix portion of the SSI subunit, while it does slightly the $\beta$-sheet portion. Difference CD of SSI* relative to that of SSI (Figure 3 ) shows two negative peaks characteristic of $\alpha$-helix and hence supports the above estimation.

\section{The Hydropathy Scale of SSI}

In order to specify which helix is associated with the conformational change induced by the hydrolysis cleavage, we applied the hydropathy index proposed by Kyte and Doolittle as a measure of hydrophobicity and hydrophilicity of the side chains of peptide bonds. ${ }^{15}$ The consecutive calculation of the average hydropathy within five amino acid residues along the primary sequence gave a hydropathy chart of amino acid side chains of the SSI subunit as shown in Figure 4. The ordinate of Figure 4 represents the hydrophobicity and hydrophilicity scales. The midpoint line indicates the grand average of the hydropathy. Secondary structure regions determined by X-ray crystallography ${ }^{3}$ are listed in the upper part of Figure 4. Peptide segments with large values of positive hydropathy are hydrophobic and correspond to the secondary structure sequences except $\beta_{2}, \beta_{5}$ strands, and $\alpha_{2}$-helix. The $\beta_{2}$ and $\beta_{5}$ strands are very short (sequence $40-42$ ) and outermost, respectively. $\mathrm{X}$-ray, ${ }^{3} \mathrm{NMR},{ }^{9,19}$ and $\mathrm{CD}^{6,8,10}$ studies have shown the $\alpha_{2}$-helix to be loose and most labile in a thermal or acid unfolding process. Side chains of amino acids composing the $\alpha_{1}$-helix region are much more hydrophilic than those composing the $\alpha_{1}$-helix.

The hydropathy index map (Figure 4) and schematic drawing of SSI structure (Figure 1) showed hydrophilic residues such as Ser 98, Asn 99, and Glu 100 to be located in the neighborhood of the scissile bond. The relative accessibilities were calculated to be $0.63,0.14$, and 0.55 for Ser 98, Asn 99, and Glu 100 in the native SSI, respectively. ${ }^{20}$ Water molecules

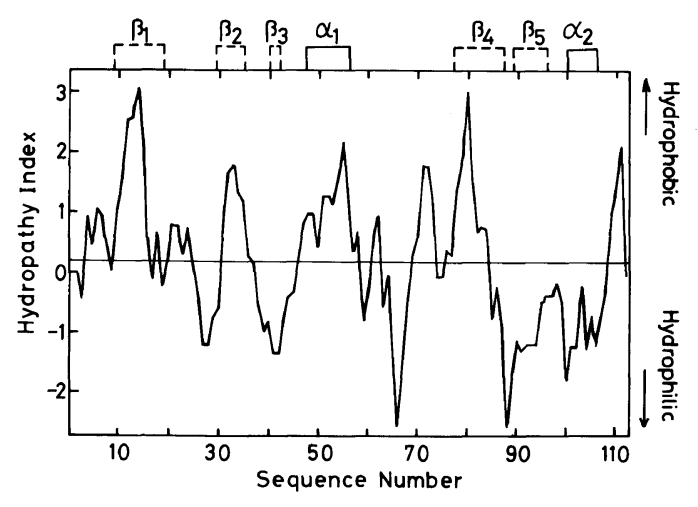

Figure 4. Map of the average hydropathy index of the side chains of amino acids obtained from the consecutive calculation within five residues along the primary sequence of SSI. Ordinate is the hydropathy scale taken from ref 15. The midpoint line indicates the grand average of the hydropathy index.

seeped through the nick will penetrate into the inside of the protein molecule through these residues and may come in the hydrophilic environment around the $\alpha_{2}$-helix. The difference CD between SSI and SSI* (dotted line in Figure 3) reflects a half-denatured state with the molten $\alpha_{2}$-helix. We actually found that Cys 71-Cys 101 bond is much more readily attacked by DTE in SSI* than in SSI. ${ }^{21}$ This fact shows that the hydrolysis at the scissile bond has a great influence on the $\alpha_{2}$-helix and Cys 71-Cys 101 bonds. The structural flexibility of the SSI outer part including the $\alpha_{2}$ helix and disulfide bond is a essential attribute of SSI. A hydrogen exchange study indicated the structural mobility of secondary structural elements at the outer part of SSI to be unusually high. ${ }^{22} \mathrm{We}$ can estimate SSI* to be much less stable than SSI.

\section{Thermal Denaturation of SSI*}

To verify the estimation, we examined the thermal denaturation of SSI* by measuring CD spectra at various temperatures. As shown in the lower part of Figure 5, an isodichroic point appeared at about $210 \mathrm{~nm}$ above $47.5^{\circ} \mathrm{C}$. The profile of the difference $\mathrm{CD}$ obtained between $25^{\circ} \mathrm{C}$ and $47.5^{\circ} \mathrm{C}$ was undefinable as 


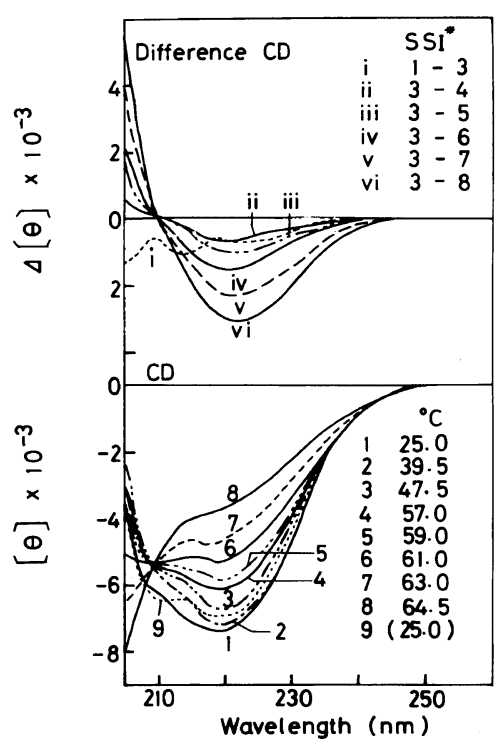

Figure 5. CD spectra of SSI* at various temperatures (lower part) and the difference CD spectra (upper part). Protein concentration was $0.125 \mathrm{mg} \mathrm{ml}^{-1}\left(5.4 \times 10^{-6} \mathrm{M}\right)$ in $0.05 \mathrm{M}$ phosphate buffer ( $\mathrm{pH} 7, \mu=0.1 \mathrm{NaCl}$ ). Optical length of the cell was $1 \mathrm{~mm}$.

shown in the upper part of Figure 5 (curve i). Curves ii-vi in Figure 5 present difference CD spectra between $47.5^{\circ} \mathrm{C}$ and various temperatures up to $64.5^{\circ} \mathrm{C}$. One negative peak and a turnover point appear at $222 \mathrm{~nm}$ and $210 \mathrm{~nm}$, respectively, indicating these difference $C D$ to be attributable to conformational change in the $\beta$-sheet. ${ }^{8}$ Difference CD profile and the isodichroic point separate the thermal denaturation process into two parts: i) step I below and ii) step II above $47.5^{\circ} \mathrm{C}$. Thermal denaturation of SSI* proceeds via the intermediate state around $47.5^{\circ} \mathrm{C}$. These characteristics are similar to the previous results ${ }^{8}$ of the intact SSI, differing only in the temperature range. Our CD study has clarified that the $\alpha_{2}$ helix unfolds in step I of the thermal de-

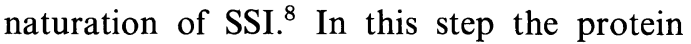
remains dimeric. An NMR signal shift of carbonyl carbon of met 103 in the $\alpha_{2}$-helix supports that the $\alpha_{2}$-helix is loosened in the intermediate state. ${ }^{19}$ Another NMR study reported a cis-trans isomerization of Ala 36-Pro

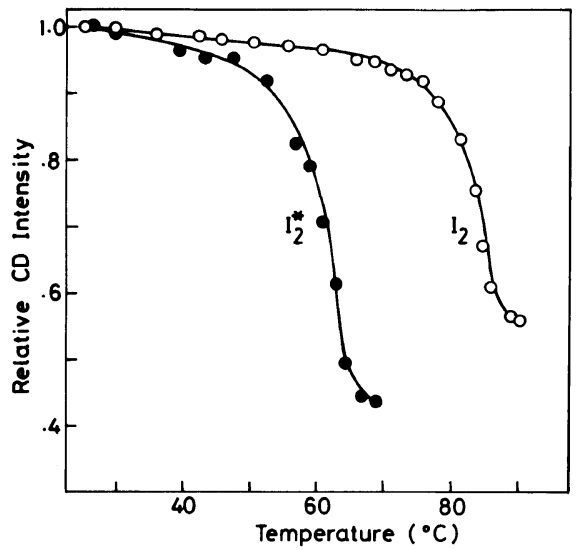

Figure 6. Temperature dependence of $\mathrm{CD}$ intensity at $220 \mathrm{~nm}$ at $\mathrm{pH} 7(\mu=0.1, \mathrm{NaCl})$. Full and empty circles indicate SSI* and SSI, respectively.

37 bond in the intermediate state. ${ }^{23}$ In step II the SSI dimer dissociates into two subunits. ${ }^{8}$ The difference CD characteristic of the $\beta$-sheet leads to this conclusion, since one SSI subunit contacts another one through $\beta$-strands. An NMR study on Arg 90 buried in the subunit interface in the native state found that the residue was exposed to solvent above $85^{\circ} \mathrm{C}^{24}$ Conformational changes similar to the parent SSI would occur in step I and step II of the thermal denaturation of SSI*.

Denaturation temperature of SSI* was estimated to be $62^{\circ} \mathrm{C}$ from temperature dependence of CD intensity at $220 \mathrm{~nm}$ (Figure 6). This temperature is about $20^{\circ} \mathrm{C}$ lower than that obtained for the intact SSI. ${ }^{8}$ Figure 6 shows the discrepancy of denaturation temperatures of SSI and SSI*. The temperature range of step II of thermal denaturation of SSI* corresponds to that of step I of thermal denaturation of SSI. Hence it was impossible to analyze the denaturation processes of SSI* and SSI simultaneously at a certain temperature to obtain the equilibrium constant between the intact and nicked species. Niekamp et $a .^{25}$ have shown the CD spectrum of the modified (nicked) soybean trypsin inhibitor (STI*) to be almost identical with that of the intact one (STI) and the stoichiometry of their 
reactions has been extensively studied. ${ }^{26,27}$ The reactive bonds of $\mathrm{SSI}^{13}$ and $\mathrm{STI}^{26}$ are located in 30-membered and 48-membered cyclic peptides linked by a disulfide bond, respectively. To explain this result, we tried to calculate average hydropathy of these two loops. The obtained values for Cys 71-Cys 101 loop of SSI and Cys 39-Cys 86 loop of STI were -0.14 and +0.40 , indicating that the loop of SSI is more hydrophilic than that of STI. Water molecules would penetrate more easily the channel of SSI* than that of STI*. At present we interprete this fact in terms of the difference between the water affinity of the cyclic peptides including the reactive sites of SSI and STI.

The equilibrium constant, $\mathrm{K}$, between the intermediate and the denatured state in step II of thermal denaturation of SSI* is given as follows,

$$
K=4 f^{2} C_{0} /(1-f)
$$

where $f$ and $C_{0}$ are the denatured fraction obtained from the denaturation profile (Figure 6) and the total protein concentration, respectively. The enthalpy change $(\Delta H)$ for this denaturation is defined as follows,

$$
\Delta H=-R(\mathrm{~d} \ln K) /(\mathrm{d} 1 / T)
$$

By substituting eq 1 into eq 2 , we obtain a linear plot of $\log \left(f^{2} / 1-f\right)$ against reciprocal temperature. From the slope of the linear plot, $\Delta H$ was estimated to be $681 \pm 33 \mathrm{~kJ} \mathrm{~mol}^{-1}$ $\left(163 \pm 8 \mathrm{kcal} \mathrm{mol}^{-1}\right)$ for step II of thermal denaturation of SSI*. Large discrepancy of $\Delta H^{\prime}$ 's of SSI* and SSI $\left(903 \pm 42 \mathrm{~kJ} \mathrm{~mol}^{-1}(216 \pm\right.$ $\left.10 \mathrm{kcal} \mathrm{mol}^{-1}\right)$ ) reflects the structure looseness induced by the hydrolytic cleavage. The corresponding $\Delta S$ 's were calculated to be $2.03 \pm 0.10 \mathrm{~kJ} \mathrm{deg}^{-1} \mathrm{~mol}^{-1}$

and

$$
\left(485 \pm 23 \mathrm{cal} \mathrm{deg}^{-1} \mathrm{~mol}^{-1}\right)
$$

$$
\begin{aligned}
& 2.54 \pm 0.12 \mathrm{~kJ} \mathrm{deg}^{-1} \mathrm{~mol}^{-1} \\
& \left(607 \pm 28 \mathrm{cal} \mathrm{deg}^{-1} \mathrm{~mol}^{-1}\right)
\end{aligned}
$$

for SSI* and SSI, respectively. The enthalpy-

\begin{tabular}{|c|c|c|c|c|}
\hline & $T_{1 / 2}$ & $\Delta H$ & $\Delta S$ & $\Delta H / \Delta S$ \\
\hline & ${ }^{\circ} \mathrm{C}$ & $\mathrm{kJ} \mathrm{mol}^{-1}$ & $\mathrm{~kJ} \mathrm{deg}^{-1} \mathrm{~mol}^{-1}$ & $\operatorname{deg}$ \\
\hline SSI* & 62 & $681 \pm 33$ & $2.03 \pm 0.10$ & $337 \pm 33$ \\
\hline SSI & 83 & $903 \pm 42$ & $2.54 \pm 0.12$ & $357 \pm 33$ \\
\hline
\end{tabular}
entropy compensation ${ }^{28}$ is confirmed by comparison of the ratios of $\Delta H$ to $\Delta S$ be-
Table I. Thermal denaturation parameters of SSI* determined by CD spectroscopy ${ }^{a}$

a In $0.05 \mathrm{M}$ phosphate buffer ( $\mathrm{pH} 7, \mu=0.1 \mathrm{NaCl}$ ). The protein concentration was $0.125 \mathrm{mg} \mathrm{m}^{-1}(5.4 \times$ $\left.10^{-6} \mathrm{M}\right)$. The values for SSI were reported previously. ${ }^{8}$

tween SSI* $(337 \pm 33 \mathrm{deg})$ and SSI $(357 \pm$ $33 \mathrm{deg}$ ). We summarized the characteristic parameters obtained for the thermal denaturation of SSI* in Table I where those for SSI are represented for comparison.

In conclusion, the hydrolysis of the scissile bond of SSI makes the loose peripheral structure of the intact SSI looser and lowers the denaturation temperature of SSI* by $20^{\circ} \mathrm{C}$ below that of SSI. But the nick does not give a remarkable effect on the central hydrophobic core comprised of extensive $\beta$-strands. Therefore, the denaturation process of SSI* remains similar to that of SSI. This is an interesting example that a slight difference in protein structure induces a large change in protein property.

Acknowledgments. The authors thank Professors S. Murao and B. Tonomura for their generous gift of the protein samples.

\section{REFERENCES}

1. T. Ikenaka, M. Odani, Y. Nabeshima, S. Sato, and S. Murao, J. Biochem., 76, 1191 (1974).

2. K. Inouye, B. Tonomura, K. Hiromi, T. Kotaka, H. Inagaki, S. Sato, and S. Murao, J. Biochem., 84, 843 (1978).

3. Y. Mitsui, Y. Satow, Y. Watanabe, S. Hirono, and Y. Iitaka, J. Mol. Biol., 131, 697 (1979).

4. K. Hiromi, K. Akasaka, Y. Mitsui, B. Tonomura, and S. Murao, Ed., "Protein Protease InhibitorThe Case of Streptomyces Subtilisin Inhibitor (SSI)," Elsevier, Amsterdam, 1985.

5. S. Murao and S. Sato, Agric. Biol. Chem., 37, 1067 (1973). 
6. T. Komiyama and M. Miwa, Polym. J., 17, 807 (1985).

7. T. Takahashi and J. M. Sturtvent, Biochemistry, 20, 6185 (1981).

8. T. Komiyama, M. Miwa, T. Yatabe, and H. Ikeda, $J$. Biochem., 95, 1569 (1984).

9. S. Fujii, K. Akasaka, and H. Hatano, J. Biochem., 88, 789 (1980).

10. T. Komiyama, A. Oomori, and M. Miwa, J. Spectrosc. Soc. Jpn., (Bunko Kenkyu), 33, 383 (1984).

11. S. Matsumori, B. Tonomura, and K. Hiromi, Abstracts of Papers, Annual Meeting of Japanese Agricultural Society, 1982, p 355.

12. H. Iwanari, Master Thesis, Kyoto University, 1984.

13. K. Omichi, N. Nagura, and T. Ikenaka, J. Biochem., 87, 217 (1980).

14. K. Akasaka. F. Hayashi, H. Hatano, B. Tonomura, H. Iwanari, and K. Hiromi, Abstracts of Papers, The Symposium on Protein Structure, 1983, p. 45 .

15. J. Kyte and R. F. Doolittle, J. Mol. Biol., 157, 105 (1982).

16. S. Sato and S. Murao, Agric. Biol. Chem., 37, 1067 (1973).

17. Y. Kanaoka, M. Machida, M. Machida, and T. Sekine, Biochim. Biophys. Acta, 371, 563 (1973).
18. S. W. Provencher and J. Glöckner, Biochemistry, 20, 33 (1981).

19. M. Kainosho and T. Tsuji, Biochemistry, 22, 6273 (1974).

20. Y. Satow, Y. Watanabe, and Y. Mitsui, J. Biochem., 88, 1739 (1980).

21. T. Komiyama, H. Masada, and M. Miwa, Abstracts of Papers, The 50th Annual Meeting of Chemical Society of Japan, 1985, p 794.

22. K. Akasaka, T. Inoue, H. Hatano, and C. K. Woodward, Biochemistry, 24, 2973 (1985).

23. H. Nagao and M. Kainosho, Abstracts of Papers, The Annual Meeting of Biochemical Society of Japan, 1984, p 632.

24. T. Tsuji, Doctoral Thesis, Tokyo University 1984.

25. C. W. Niekamp and M. Laskowski, Jr., Abstracts of Papers, the 158th Meeting of American Chemical Society, New York, N. Y., (1969), Biology No. 273.

26. C. W. Niekamp, H. F. Hixson, Jr., and M. Laskowski, Jr., Biochemistry, 8, 16 (1969).

27. P. Desnuelle, H. Neurath, and M. Ottesen (Ed.), "Structure-Function Relationship of Proteolytic Enzymes," Munksgaard, Copenhagen, 1970, pp. 89-101.

28. R. Lumry and S. Rajender, Biopolymers, 9, 1125 (1970). 